\title{
The effect of medium of instruction on undergraduate student learning style in video- based learning
}

\author{
Nives Mikelic Preradovic, Tomislava Lauc, Ida Panev
}

\begin{abstract}
The study aims to assess the impact of interactive and linear video on learning effectiveness of undergraduate informatics students with different predominant learning styles (visual, aural, read/write and kinesthetic style). The students in different treatment groups (learning with interactive vs. linear video) have achieved very good learning outcomes and those who used interactive videos achieved better learning outcomes. The impact of the kinesthetic predominant learning style on learning with interactive videos was not evident, i.e. the predominant learning style of the undergraduate informatics students did not affect their success, regardless of the type of video.
\end{abstract}

Keywords-demonstration video, instructional video, interactive video, linear video, multimedia learning, VARK sensory modality, video-based learning.

\section{INTRODUCTION}

$\mathrm{I}$ nstructional videos are commonly used as supplemental materials to enhance learning during lectures or training, created to lead learners towards a specific action and enabling them to perform the tasks without video support [1], [2], [3], [4]. Video allows learners to view realistic scenes and visualize dynamic processes that are complex or difficult to verbally describe, as well as costly or dangerous to perform. It enables the demonstration of techniques or skills, simulation of actual events or experiments and illustration of ideas using a slow-motion or fast camera. It also allows for combining different symbolic systems into coherent multimedia messages. Video is a dynamic resource that can be used for learning, enabling visualization and analysis while facilitating understanding of the presented educational materials. It particularly attracts individuals with multimodal sensory preferences because of the potential to transmit information through both the auditory and the visual channel at the same time.

The interest for instructional videos is gaining momentum

Nives Mikelic Preradovic is a full professor at the Department of Information and Communication Sciences, Faculty of Humanities and Social Sciences, University of Zagreb, Croatia. E-mail: nmikelic@ ffzg.hr

Tomislava Lauc is a full professor at the Department of Information and Communication Sciences, Faculty of Humanities and Social Sciences, University of Zagreb, Croatia. E-mail: tlauc@ffzg.hr

Ida Panev is a Lecturer at the Polytecnic of Rijeka, Croatia. E-mail: ipanev@ veleri.hr due to the advancement of new forms of online education, such as massive open online courses (MOOCs) that aim to counter the rising cost of higher education [5]. Millions of students are learning through videos reproduced through various platforms (e.g. YouTube, Coursera, EdX, Udacity, etc.). The most common types of videos that are distributed through MOOCs are live streaming lectures and instructional videos.

Although MOOCs have many advantages, a high dropout rate from these online courses was observed over the past few years. According to [6], although thousands of attendees are enrolled in these courses, only a small number completes a course.

Nawrot and Doucet [7] state that among the five main reasons for dropping out of MOOCs is the fact that courses do not actively involve their students or that they are not attractively presented. If video in MOOCs is used as a linear medium designed to deliver a lecture, it turns learners into passive viewers, depriving them of opportunities for constructive learning.

According to [8], linear (non-interactive) videos used in the educational process can expose the individual to a new concept in an appropriate way, but they do not produce learning. Barba [8] considers such videos to be equivalent to traditional ex-cathedra teaching. In addition to traditional lectures, they are convenient since an individual does not have to be at the same time in the same place with a lecturer and since it is possible to control the playback of the video (start, stop and skim) and navigate the videos in one's own pace.

But, learners need to become familiar with new concepts in the educational process in various ways, communicate ideas and problems, and be active at each stage of the information acquisition process, leaning into the experience, choosing their own adventure, instead of leaning back and being passive learners. Barba [8] claims that linear videos, as well as traditional lectures, do not encourage learning, because learning is achieved by actively involving an individual in the learning process. Interactivity that provides in-context clues and enables learners to immediately take the action that a lecturer wants them to take represents a more conversational and more engaging way of learning, satisfying learners' needs, saving their time and creating more effective overall user experience.

Enrolling in a MOOC where only linear videos are used, students quickly lose interest and motivation and consequently 
drop out [6]. In linear videos, the lack of interactivity affects the ability to meet the educational needs of individuals, thereby reducing their educational success [9].

The ultimate goal of using videos in the educational process should be to create a multisensory learning environment that can enhance students' ability to retain information [10]. But, the research results about the effect of medium of instruction (interactive vs. non-interactive video) on the educational success of learners with different predominant learning styles show a lack of consistency.

Some of the existing studies indicate that student's predominant learning style represents a very important factor in learning since learning styles had significant effects on students' knowledge acquisition in two different learning environments: online instruction and traditional instruction [11], [12]. Both authors reported that students with a particular learning style performed better than students with other learning preferences in the online setting.

On the other hand, [13], [14], [15], [16] reported that learning styles did not influence students' mean test scores and that learning styles had no statistically significant effect on learning performance in any of the two instructional methods (online instruction vs. traditional instruction).

Some researchers suggest that instructional design should meet different learning styles in course design and delivery [17]. Other studies discovered that visual, auditory, and kinesthetic learners show similar knowledge gains when learning using video and that all of them prefer similar elements: clear instruction, high visual and audio quality and an easy-to-follow strategic video flow [18].

Therefore, this paper focuses on assessing the impact of interactive and non-interactive video on the learning effectiveness of students with different predominant learning styles.

The remainder of this paper is organized as follows. In the next section, the literature review about instructional design that maximizes students' learning opportunities and addresses their learning needs is provided. The following section describes the methodology of the study. After it, the experimental results obtained by the proposed method are provided. Finally, the paper concludes with some suggestions and remarks.

\section{LITERATURE REVIEW}

\section{A. Cognitive theory of multimedia learning}

According to [19] there are three assumptions underlying the cognitive theory of multimedia learning: 1) humans possess separate information processing channels (auditory and visual), 2) humans are limited in the amount of information that can be processed in each channel at one time (similar to Sweller's notion of Cognitive Load) and 3) humans engage in active learning by attending to relevant incoming information, organizing selected information into coherent mental representations, and integrating mental representations with other knowledge.

Based on these assumptions, The Cambridge handbook of multimedia learning [20] offers principles for 1) managing essential processing in multimedia learning: segmenting, pretraining, and modality principles [21], 2) principles for reducing extraneous processing in multimedia learning: coherence, signaling, redundancy, spatial contiguity, and temporal contiguity principles [22] and 3): principles based on social cues in multimedia learning: personalization, voice, image, and embodiment principles [20].

\section{B. Demonstration-based-training}

Demonstration-based-training (DBT) is an approach to instructional video design based on four fundamental processes involved in observational learning distinguished by [23] attention, retention, (re)production and motivation. In DBT, a dynamic example of performance is complemented with instructional features [24], [25].

\section{Attention cueing}

Spatial attention can be attracted by a variety of visual cues or signals, such as movement, color or labels.

Signaling reduces the possibility of potential cognitive overload because it requires less cognitive resources to find relevant information. In order to prevent an individual from focusing on irrelevant information within the educational material, it is necessary to use the signaling that directs cognitive processing to the relevant information [26]. Thus, the principle of signaling is extremely important during the selection of the relevant incoming information from the video, as it enables the organization and integration of the relevant information [26].

The purpose of signaling is not to add new information to the presented material, but rather to direct cognitive processes to the content that is relevant [27]. Signaling serves as a cognitive guide that helps learners to create meaning by reducing the cognitive load or by reducing the possibility of cognitive overload.

Cueing has been shown to reduce cognitive load, guide the learner's attention and foster learning [26], [28], [29], [30], [31], [32], especially in learners with low to medium priorknowledge [33]. But, there are also studies that reveal no significant positive effects of attention cueing on comprehension [34], [35].

\section{Retention}

Mayer [19] writes about the processing of relevant and irrelevant information within the cognitive learning theory. Processing of relevant information involves selecting important information as a result of well-formed instructional design of educational material, while processing of irrelevant information (in-formation not related to learning outcomes) increases cognitive load and leads to inefficient learning [36].

Retention (the learner's ability to retrieve and execute an action stored in the memory) includes organizing relevant information into coherent mental representations which are then integrated with previous knowledge.

In order to support the learner's retention, [25] propose the inclusion of temporal cueing (brief pauses of 2-5 seconds), allowing learners to grasp what they have observed and to integrate the new information with previous knowledge.

Empirical evidence of the retention-supportive role of temporal cueing that is the result of segmentation in instructional animations (videos, computer animations) has 
been reported by [37], [38], [39], [40].

\section{Production and Motivation}

Production can be defined as the learner's capacity to execute the procedural steps required to correctly complete a task [23].

An instructional measure that can enhance production is the inclusion of practice since it stimulates reflection and consolidates learning [41]. Unfortunately, the research about the effects of practice with videos for software training is rare and with mixed results. The researchers have failed to find evidence of the contribution of practice for learning if practice was followed by video or video was followed by practice [41]. On the other hand, some experiments [42] show significant learning gains in procedural knowledge if a practice was implemented directly after the video (there were no effects on the declarative knowledge). Furthermore, it is recommended to include interactivity in the video to ensure that procedural knowledge is attained if a practice is not a part of the learning environment [42].

Motivation refers to the processes that account for an individual's intensity, direction, and persistence of effort toward attaining a goal [43]. Motivation of learners can be enhanced by anchoring of the tools in the task domain, according to the second principle of minimalist instruction. This principle specifically calls for the use of tasks that are core tasks for the learners who are becoming acquainted with new tools or applications that are going to be used in their everyday life.

The authors of this paper believe that learning progress can be achieved by applying the principles of multimedia learning and demonstration-based-training (DBT) in instructional video design, but also through interactivity, i.e. if interactivity is part of the video, which will be further elaborated in the rest of the paper.

\section{VARK sensory model}

VARK is a sensory model developed by New Zealand professor Neil Fleming [44]. VARK is an acronym for Visual: Visual, Aural, Read/write and Kinesthetic sensory modalities of an individual used to learn information. Before Fleming's model, a VAK model was in use [45], and Fleming divided the visual dimension into two parts: the symbolic part (visual $\mathrm{V}$ ) and the textual part (read/write - R). There are also senses of touch and taste, but they are generally not used to acquire the educational content.

Fleming [46] defines a learning style as the characteristics of an individual and the preferred way of collecting, organizing, and understanding information. The learning style can be represented with a sensory modality. In other words, sensory modality can be described as a learning style. Determining the learning style of students enhances the performance of the learning process [47].

\section{VARK modalities}

If a person has visual primary modality, then he/she prefers data arranged in mental maps, network diagrams, charts and graphs, flowcharts, hierarchies, and symbols. In short, such a person prefers learning by using visually presented material than written material. A person with a primary visual modality will successfully acquire written information using underlining, highlighting, different colors, markers, symbols, patterns, and shapes.

Those having aural primary modality learn best by listening, participating in group discussions, listening to the radio or audio recordings, talking aloud about a topic, reading and rehearsing aloud, using a voice recorder and mobile audio communication capabilities.

If a person has reading primary modality, then he/she prefers the written information - he/she learns by reading and writing. Many teachers and students have preferences for this modality, which emphasizes the textual input and output: manuals, reports, essays, and written assignments. Individuals with the reading primary modality often depend heavily on PowerPoint, the Internet, various lists, diaries, dictionaries, thesauruses, citations, and all other written information.

Those having kinesthetic primary modality prefer to learn using their own experience and practice, through participation in real or simulated events. In other words, such individuals acquire information through concrete personal experiences, examples, practice, simulations, demonstrations, video materials, case studies, projects, experiments and the application of knowledge.

\section{VARK questionnaire}

The VARK questionnaire is an instrument for determining the learning preference of a subject using the VARK sensory model. This questionnaire measures the presence of each of the four sensory modalities in the subjects. Each individual can have one, two, three or four modalities. According to research [46], it is rarely the case that an individual possesses only one sensory preference. The questionnaire is designed in such a way that one or more answers to each question can be selected. Individuals who select multiple responses fall into the multimodal group.

According to [46], there are two types of multimodal persons. VARK type 1 are individuals who switch from one modality to another depending on the context. For example, if they must learn a law, they will express their reading preference, and if they have to look at a demonstration of certain skills, then their kinesthetic preference will be expressed. Such types of individuals will have two, three or four equally represented sensory preferences in the results of the VARK questionnaire. VARK Type 2 are individuals who will not be satisfied until they have input or output information in each of their preferred modalities. Such individuals will need more time to gather information in all the preferred ways, but as a result, they will have a deeper and broader understanding. Their decision-making and learning can be better thanks to a breadth of understanding.

Using a VARK questionnaire, [48] pointed to a link between kinesthetic learning style and the choice of multimedia learning resources. In more detail, in Byrne's study, $47.05 \%$ of respondents identified as kinesthetic types chose interactivity as their preferred method of learning.

In our study, we used the VARK questionnaire to test the hypothesis that kinesthetic types of learners prefer interactive 
video over passive video.

\section{METHOD}

The study aims to assess the impact of interactive and noninteractive video on the learning effectiveness of students with different predominant learning styles.

The instructional videos (both non-interactive and interactive) aimed to teach undergraduate informatics students formatting in Microsoft Word 2010. They were divided into six chapters and designed according to the principles of Cognitive Theory of Multimedia Learning [19] and Demonstration-based-training [23]. Special attention was paid to the implementation of attention, retention, (re)production and motivation in videos.

There were 52 students who were randomly assigned to two groups: one group learned using non-interactive video, while the other group used interactive video with the same learning content. The initial VARK questionnaire was used to collect data about students' predominant sensory modality and demographic data. Pre-test and post-test assessed learning before and after each video chapter. The questions in both tests were different but tested the same content.

Our research question is whether students with a kinesthetic learning style achieve better learning results if they learn from either interactive or demonstration video than students with other learning styles.

\section{RESUlTS AND DisCUSSION}

In each of the 15 questions of the VARK questionnaire, 4 answers were offered, each was related to one sensory modality. Respondents were able to select multiple answers to each question. The analysis of the questionnaire brought us to the following conclusions:

- the majority of respondents (55.77\%) had a kinesthetic learning style: $40.38 \%$ of those had predominant kinesthetic style, while $15.38 \%$ were a combination of several styles: kinesthetic and aural (5.77\%), kinesthetic and reading (7.69\%), and kinesthetic, aural and reading learning style (1.92\%);

- 36.54\% of students had the aural modality: $26.92 \%$ of those had predominant aural style, while $5.77 \%$ were a combination of aural and kinesthetic, and $1.92 \%$ were a combination of aural and visual learning style;

- $21.15 \%$ of students had a reading learning style: $9.62 \%$ of those had predominant reading style, $7.69 \%$ were found to be a combination of reading and kinesthetic style, while $1.92 \%$ was a combination of reading, kinesthetic and aural style, and $1.92 \%$ was a combination of reading and visual style;

- the least represented learning style was the visual one since only $7.69 \%$ of respondents belonged to this style.

Out of these, $3.85 \%$ were predominantly visual types, $1.92 \%$ were a combination of visual and reading style, and $1.92 \%$ were visual and aural type.

Of the 52 respondents, $42 \quad(80.77 \%)$ had a single predominant modality (Table 1$)$ :

- visual: 2 respondents (3.85\%);

- aural: 14 respondents (26.92\%);
- reading: 5 respondents (9.62\%);

- kinesthetic: 21 respondents (40.38\%).

Among the respondents, $19.23 \%$ had multimodal preferences, $17.31 \%$ of which were bimodal (combinations: Table 1. Sensory modalities of students (VARK)

\begin{tabular}{|c|c|c|}
\hline & $\mathrm{n}$ & \\
\hline V & 2 & \multirow{3}{*}{ Unimodal } \\
\hline A & 14 & \\
\hline R & 5 & \multirow{3}{*}{ Bimodal } \\
\cline { 1 - 2 } K & 21 & \\
\hline RK & 4 & \\
\hline VR & 1 & Trimodal \\
\hline VA & 1 & 3 \\
\hline AK & 1 & \\
\hline ARK & \multicolumn{2}{|c|}{ Total = 52 students } \\
\hline \multicolumn{2}{|c|}{}
\end{tabular}

RK, VR, VA, AK), and $1.92 \%$ were trimodal (combination: ARK).

The frequency of VARK modalities (unimodal, bimodal and trimodal) did not differ between the groups learning

Table 2. VARK characteristics of two groups of respondents with respect to video type (linear/interactive)

\begin{tabular}{|c|c|c|c|}
\hline & $\begin{array}{c}\text { All students } \\
(\mathrm{n}=52)\end{array}$ & $\begin{array}{c}\text { Linear } \\
\text { group } \\
(\mathrm{n}=24)\end{array}$ & $\begin{array}{c}\text { Interactive } \\
\text { group } \\
(\mathrm{n}=28)\end{array}$ \\
\hline VARK modality: & & & \\
Unimodal, n (\%) & $42(81 \%)$ & $18(75 \%)$ & $24(86 \%)$ \\
Bimodal, n (\%) & $9(17 \%)$ & $5(21 \%)$ & $4(14 \%)$ \\
Trimodal, n (\%) & $1(2 \%)$ & $1(4 \%)$ & $0(0 \%)$ \\
\hline Kinestethic modality: & & & \\
Unimodal, n (\%) & $21(40 \%)$ & $11(46 \%)$ & $10(36 \%)$ \\
Multimodal, n (\%) & $8(15 \%)$ & $5(21 \%)$ & $3(11 \%)$ \\
Non-kinestethic, n (\%) & $23(44 \%)$ & $8(33 \%)$ & $15(54 \%)$ \\
\hline
\end{tabular}

through interactive and non-interactive videos.

Also, the groups were approximately equal regarding the frequency of kinesthetic learning styles (unimodal, multimodal, non-kinesthetic) (Table 2).

The results of the pre-tests and post-tests and comparison of subjects with regard to the type of video used for learning (interactive/non-interactive) and VARK type (kinesthetic / non-kinesthetic) are shown in the Table3 and Table4.

The group of kinesthetic subjects includes subjects who had a unimodal predominant kinesthetic learning style, while multimodal types of learners with kinesthetic style as one of the preferred styles were excluded from the comparison. The group of non-kinesthetic subjects included all subjects who had unimodal or multimodal learning preferences in which kinesthetic preference was absent. Each test for each video chapter is treated as an equal unit, so the success rate for each test is taken into account. Thus, a composite variable containing pre-test scores was created for all six video 
Table 3. Descriptive statistics of pre-test score regarding VARK modality and group treatment (linear /interactive video)

\begin{tabular}{|l|l|l|l|}
\hline Learners & Means & SD & N \\
\hline Kinesthetic interactive & 38,39 & 20,04 & 16 \\
\hline $\begin{array}{l}\text { Kinesthetic } \\
\text { linear }\end{array}$ & 42,24 & 22,79 & 13 \\
\hline Kinesthetic & 40,12 & 21,01 & 29 \\
\hline & & & \\
\hline $\begin{array}{l}\text { Non-Kinesthetic } \\
\text { interactive }\end{array}$ & 53,04 & 13,67 & 8 \\
\hline $\begin{array}{l}\text { Non-Kinesthetic } \\
\text { linear }\end{array}$ & 37,00 & 17,12 & 15 \\
\hline Non-Kinesthetic & 42,57 & 17,52 & 23 \\
\hline & & & 24 \\
\hline Interactive & 43,27 & 19,19 & 24 \\
\hline Linear & 39,43 & 19,75 & 28 \\
\hline Total & 41,20 & 19,40 & 52 \\
\hline
\end{tabular}

Table 4. Descriptive statistics of post-test score regarding VARK modality and group treatment (linear /interactive video)

\begin{tabular}{|l|l|l|l|}
\hline Learners & Means & SD & N \\
\hline Kinesthetic interactive & 84,16 & 18,62 & 16 \\
\hline $\begin{array}{l}\text { Kinesthetic } \\
\text { linear }\end{array}$ & 91,83 & 9,64 & 13 \\
\hline Kinesthetic & 87,64 & 15,51 & 29 \\
\hline $\begin{array}{l}\text { Non-Kinesthetic } \\
\text { interactive }\end{array}$ & 82,93 & 18,77 & 8 \\
\hline $\begin{array}{l}\text { Non-Kinesthetic } \\
\text { linear }\end{array}$ & 85,75 & 15,90 & 15 \\
\hline Non-Kinesthetic & 84,77 & 16,58 & 23 \\
\hline & & 18,27 & 24 \\
\hline Interactive & 83,75 & 13,49 & 28 \\
\hline Linear & 88,57 & 15,90 & 52 \\
\hline Total & 86,35 & & \\
\hline
\end{tabular}

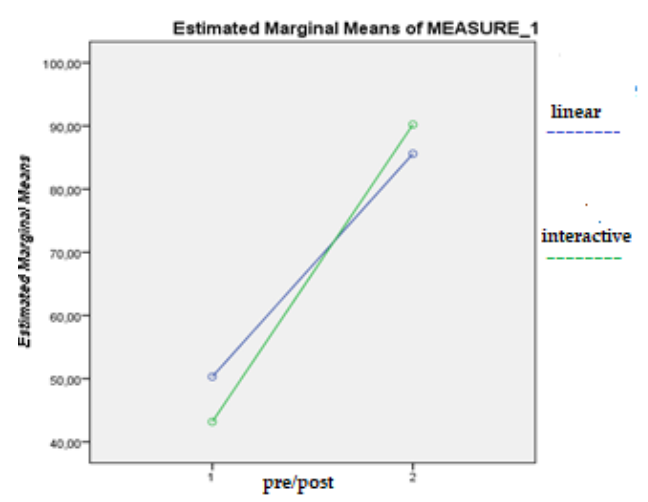

Fig. 1 interaction of two-time points and medium of instruction chapters and a composite variable containing post-test scores, was made for all six video chapters.

Taking into consideration the confirmation that data for each sample is normally distributed, we performed a repeatedmeasures ANOVA.

Tables 3 and 4 show the means and standard deviations of pretest scores and posttest scores of students in different treatment groups and in regard to different modalities.

The results have shown that mean score for pre-test and post-test differed statistically significantly $(F(1,48)=$ 280,829, $\mathrm{p}=0.000$ ).

Also, there is an interaction for level of activity considering interactive video vs. non-interactive video $(\mathrm{F}(1,48)=4,770$, $\mathrm{p}=0,034)$, although the results of a post-hoc Tukey test were not significant.

According to the results $(\mathrm{F}(1,48)=2,098, \mathrm{p}=0,154)$, it is not proved that the learning preference of the student affects the student's success.

The students have achieved very good learning outcomes, but those who used interactive videos achieved better learning outcomes (Fig.1).

In this study, we revealed that kinesthetic learning preference does not affect the student's success.

Our results are in line with studies [13], [14], [15], [16] that claim that there are no significant differences in learning achievement based on learning style preferences. We did not find evidence to support the results of the studies [11], [12] that argue that there are significant differences in learning outcomes and that these variations depend on learning style preferences.

Furthermore, our study revealed the importance of instructional strategy which implements active learning and engages the learner in the learning environment, since students who used interactive videos achieved better learning progress. Although the group of kinesthetic learners included only those with unimodal kinesthetic style, while multimodal types of learners with kinesthetic style as one of the preferred styles were excluded, the impact of the kinesthetic preference on learning with interactive videos was not evident. However, if the influence of a learning style is questionable, the importance of a useful learning tool, based on instructional design principles, still remains indisputable.

\section{CONCLUSION}

The study compared six units of the same course on Microsoft Word 2010 that were taught to undergraduate informatics students parallelly using interactive and noninteractive videos. Even though the students in both groups (interactive /non-interactive) were not preselected, the demographics of age and predominant sensory modality showed no significant differences between the two groups.

To ascertain the learning styles of the students, the VARK instrument was used. Approximately two-thirds of each group were unimodal learners, while unimodal kinesthetic learners in both groups were the most represented. The result of the study reveals that the learning preference of the undergraduate informatics students does not affect the student's success, regardless of the type of video. It supports the previous research [18] on equal learning success of students with different predominant learning styles.

However, both types of videos had a positive effect on the learning process, and students have achieved very good 
learning outcomes, proving the instructional effectiveness of both videos. Also, students who used interactive videos achieved better learning outcomes.

The importance of multimedia instructional design principles (i.e. the application of the principles of Cognitive Theory of Multimedia Learning [19] and demonstrationbased-training principles [25]) proved to be vital regardless of the medium of instruction.

Although there is a lack of significant differences in learning outcomes considering learning preferences, educators can still facilitate knowledge acquisition considering different learning strategies. If educators possess the awareness of the importance of instructional design that can enhance student learning, engagement through interactivity remains more optional.

We expect these findings to help higher-education teachers understand how to design high-quality videos to maximize student learning success.

\section{REFERENCES}

[1] F. Grabler, M. Agrawala, W. Li, M. Dontcheva, and T. Igarashi, "Generating Photo Manipulation Tutorials by Demonstration," in Proc. SIGGRAPH, pp.1-669, 2009.

[2] H. Van der Meij, J. Karreman, and M. Steehouder," Three Decades of Research and Professional Practice on Printed Software Tutorials for Novices," Technical Communication, vol. 56, no. 3, pp. 265-292, 2009.

[3] T. Lucas, and A.R. Ruslan, "Designing Instructional Animation for Psychomotor Learning - A Conceptual Framework,” in Proc. Seventh International Conference on Computer Supported Education, pp. 313318, 2015, doi: 10.5220/0005477303130318

[4] R.H Kay, "Developing a Framework for Creating Effective Instructional Video Podcasts," International Journal of Emerging Technologies in Learning, vol. 9, no. 1, p.22-30, 2014.

[5] J. Baggaley, "The Sudden Revival of Educational Video," in Proceedings of the 2013 IEEE 63rd Annual Conference International Council for Education Media, ICEM 2013, pp. 1-6, 2013.

[6] D.F.O. Onah, J. Sinclair, and R. Boyatt, "Dropout Rates of Massive Open Online Courses: Behavioural Patterns," in Proc. Sixth International Conference on Education and New Learning Technologies (EDULEARN '14), pp. 5825-5834, 2014.

[7] I. Nawrot, and A. Doucet, "Building Engagement for MOOC Students Introducing Support for Time Management on Online Learning Platforms," in Proceedings of the companion publication of the 23rd International conference on world wide web companion, pp. 1077-1082, 2014.

[8] L. Barba, "Why My MOOC Is Not Built on Video," Class Central, https://www.class-central.com/report/why-mymooc-is-not-built-onvideo/, 2015 (URL link: 2019)

[9] D. Zhang, L. Zhou, R.O. Briggs, and J.F.Jr. Nunamaker, "Instructional Video In E-Learning: Assessing the Impact of Interactive Video on Learning Effectiveness," Information \& Management, vol. 43, no. 1, pp. 15-27, 2006, doi:10.1016/j.im.2005.01.004

[10] M.R. Syed, "Diminishing the Distance in Distance Education", IEEE MultiMedia, vol. 8, no. 3, pp. 18-21.

[11] N. Bozionelos, "Psychology of Computer Use: XLIV: Computer Anxiety and Learning Style," Perception and Motor Skills, vol. 84, pp. 753-754, 1997.
[12] S. Rakap, "Impacts of Learning Styles and Computer Skills on Adult Students' Learning Online," The Turkish Online Journal of Educational Technology, vol. 9, no. 2, pp. 14-21, 2010.

[13] J.P. Buerck, T. Malmstrom, and E. Peppers, "Learning environments and learning styles: Non-traditional student enrollment and success in an Internet-based versus a lecture-based computer science course," Learning Environments Research, vol. 6, pp. 137-155, 2003.

[14] Y. Liu, "A Comparative Study of Learning Styles between Online and Traditional Students," Journal of Educational Computing Research, vol. 37, no. 1, pp. 41-63, 2007.

[15] N.Z. Zaharis, "The Impact of Learning Styles on Student Achievement in a Web-Based versus an Equivalent Face-to-Face Course", College Student Journal, vol. 44, no. 3, pp. 591-597, 2010.

[16] R.N. Harris, W.O. Dwyer, and F.C. Leeming, "Are Learning Styles Relevant in Web-Based Instruction?" Journal of Educational Computing Research, vol. 29, pp. 13-38, 2003.

[17] S.H. Zhong, Y.H. Li, Y. Liu, and Z.Q. Wang, "A Computational Investigation of Learning Behaviors in Moocs," Comput. Appl. Eng. Educ. vol. 25, no. 5, pp. 693-705, 2017.

[18] N.M. Noor, M. Aini, and N. Izzati, "Video Based Learning Embedded with Cognitive Load Theory: Visual Auditory and Kinaesthetic Learners 'Perspectives", in Proc. 2014 International Conference on Teaching and Learning in Computing and Engineering, pp. 58-63, 2014.

[19] R.E. Mayer, ed., The Cambridge Handbook of Multimedia Learning. New York, NY, US: Cambridge University Press, 2005, pp. 34-35. doi:10.1017/CBO9780511816819

[20] R.E. Mayer, "Principles Based on Social Cues in Multimedia Learning: Personalization, Voice, Image, And Embodiment Principles," in The Cambridge handbook of multimedia learning, R.E. Mayer, ed., New York, NY, US: Cambridge University Press, pp. 345-368, 2014, doi:10.1017/CBO9781139547369.017

[21] R.E. Mayer, and C. Pilegard, "Principles for Managing Essential Processing in Multimedia Learning: Segmenting, Pre-Training, And Modality Principles," in The Cambridge handbook of multimedia learning, R.E. Mayer, ed., New York, NY, US: Cambridge University Press, pp. 316-344, 2014.

[22] R.E. Mayer, and L. Fiore, "Principles for Reducing Extraneous Processing in Multimedia Learning: Coherence, Signaling, Redundancy, Spatial Contiguity, And Temporal Contiguity Principles," in The Cambridge handbook of multimedia learning, R.E. Mayer, ed., New York, NY, US: Cambridge University Press, pp. 279-315, 2014.

[23] A. Bandura, Social Foundations of Thought and Action: A Social Cognitive Theory. Englewood Cliffs, NJ: Prentice-Hall, 1986.

[24] R. Grossman, E. Salas, D. Pavlas, and M.A. Rosen, "Using Instructional Features to Enhance Demonstration-Based Training in Management Education," Academy of Management Learning \& Education, vol. 12, no. 2, pp. 219-243, 2013, doi:10.5465/amle.2011.0527

[25] H. van der Meij, and J. van der Meij, "Demonstration-Based Training (DBT) In the Design of a Video Tutorial for Software Training," Instructional science, vol. 44, no. 6, pp. 527-542, 2016, doi: 10.1007/s11251-016-9394-9

[26] T. van Gog, "The Signaling (or Cueing) principle in multimedia learning," in The Cambridge Handbook of Multimedia Learning, R.E. Mayer, ed., New York, NY, US: Cambridge University Press, pp. 263278, 2014, doi: 10.1017/CBO9781139547369.014

[27] P. D. Mautone, and R.E. Mayer, "Signaling as A Cognitive Guide in Multimedia Learning," Journal of Educational Psychology, vol. 93, no. 2, pp. 377-389, 2001.

[28] J.M. Boucheix, and R.K. Lowe, "An Eye Tracking Comparison of External Pointing Cues and Internal Continuous Cues in Learning with 
Complex Animations," Learning and Instruction, vol. 20, pp. 123-135, 2010, doi: 10.1016/j.learninstruc.2009.02.015

[29] B.B de Koning, H.K. Tabbers, R.M. Rikers, and F. Paas, "Attention Cueing as a Means to Enhance Learning from an Animation," Applied Cognitive Psychology, vol. 21, pp. 731-746, 2007, doi: 10.1002/ acp.1346

[30] B.B de Koning, H.K. Tabbers, R.M. Rikers, and F. Paas, "Learning by Generating Vs. Receiving Instructional Explanations: Two Approaches to Enhance Attention Cueing in Animations," Computers \& Education, vol. 55, pp. 681-691, 2010, doi: 10.1016/j.compedu.2010.02.027

[31] R. E. Mayer, \& L. Fiorella, "Principle for Reducing Extraneous Processing in Multimedia Learning: Coherence, Signaling, Redundancy, Spatial Contiguity, And Temporal Contiguity Principles," in The Cambridge handbook of multimedia learning, R.E. Mayer, ed., New York, NY, US: Cambridge University Press, pp. 279-315, 2014.

[32] F. X. Wang, Z.H. Duan, and Z.K. Zhou, "Attention Guidance in Multimedia Learning: The Role of Cueing," Advances in Psychological Science, vol. 21, pp. 1430-1440, 2013, doi: 10.3724/SP.J.1042.2013 .01430

[33] J. Richter, K. Scheiter, and A. Eitel, "Signaling Text-Picture Relations in Multimedia Learning: A Comprehensive Meta-Analysis," Educational Research Review, vol. 17, pp. 19-36, 2016, doi: 10.1016/j.edurev.2015.12.003

[34] S. Kriz, and M. Hegarty, "Top-Down and Bottom-Up Influences on Learning from Animations," International Journal of Human Computer Studies, vol. 65, pp. 911-930, 2007, doi: 10.1016/j.ijhcs.2007.06.005

[35] I.T. Skuballa, R. Schwonke, and A. Renkl, "Learning from Narrated Animations with Different Support Procedures: Working Memory Capacity Matters," Applied Cognitive Psychology, vol. 26, pp. 840-847, 2012, doi: 10.1002/acp.2884

[36] J. Sweller, P. Ayres, and S. Kalyuga, Cognitive Load Theory. New York: Springer, 2011.

[37] B.S. Hasler, B. Kersten, and J. Sweller, "Learner Control, Cognitive Load And Instructional Animation," Applied Cognitive Psychology, vol. 21, pp. 713-729, 2007, doi:10.1002/acp.1345.

[38] H. Hassanabadi, E.S. Robatjazi, and A.P. Savoji, "Cognitive Consequences of Segmentation and Modality Methods in Learning from Instructional Animations," Social and Behavioral Sciences, vol. 30, pp. 1481- 1487, 2011, doi: 10.1016/j.sbspro.2011.10.287

[39] R. Moreno, and R.E. Mayer, "Interactive Multimodal Learning Environments," Educational Psychology Review, vol. 19, pp. 309-326, 2007, doi:10.1007/s10648-007-9047-2

[40] I.A.E. Spanjers, T. van Gog, P. Wouters, and J.J.G. van Merrienboer, "Explaining the Segmentation Effect in Learning from Animations: The Role of Pausing and Temporal Cueing," Computers \& Education, vol. 59, pp. 274-280, 2012, doi: 10.1016/j.compedu.2011.12.024

[41] H. van der Meij, I. Rensink, and J. van der Meij, "Effects of Practice with Videos for Software Training," Computers in Human Behavior, (Special issue), pp. 439-445, 2018, doi: 10.1016/j.chb.2017.11.029

[42] A. Ertelt, "On-Screen Videos as An Effective Learning Tool: The Effect of Instructional Design Variants And Practice On Learning Achievements, Retention, Transfer, And Motivation," PhD dissertation, Freiburg (Breisgau), Germany, 2007.

[43] S. Robbins, and T. Judge, Organizational Behavior, Pearson Prentice Hall, 2009, pp.75-76.

[44] N.D. Fleming, and C. Mills, "Not Another Inventory, rather a Catalyst for Reflection," To Improve the Academy, vol. 11, pp. 137-155, 1992.

[45] W. Barbe, S. Burke, H. Raymond, and M.N. Milone, Teaching Through Modality Strengths: Concepts and Practices, Columbus, Ohio: ZanerBloser, 1979.
[46] N. Fleming, Teaching and Learning Styles: VARK Strategies, Christchurch, New Zealand: N.D. Fleming, 2011.

[47] M. Abdullah, A. Alqahtani, J. Aljabri, R. Altowirgi, and R. Fallatah, "Learning Style Classification Based on Student's Behavior in Moodle Learning Management System," Transactions on Machine Learning and Artificial Intelligence vol. 3, no. 1, 2015.

[48] D. Byrne, A Study of Individual Learning Styles and Educational Multimedia Preferences an Experiment Using Self- Directed Online Learning Resources, School of Computer Applications, Ireland: Dublin City University Press, 2002. 